Article

\title{
Predictors of Patient-Reported Incontinence at Adjuvant/Salvage Radiotherapy after Prostatectomy: Impact of Time between Surgery and Radiotherapy
}

\author{
Fernando Munoz ${ }^{1}\left(\mathbb{D}\right.$, Giuseppe Sanguineti ${ }^{2}$, Andrea Bresolin ${ }^{3}$, Domenico Cante ${ }^{4}\left(\mathbb{D}\right.$, Vittorio Vavassori $^{5}$, \\ Justina Magdalena Waskiewicz ${ }^{6}$, Giuseppe Girelli ${ }^{7}$, Barbara Avuzzi ${ }^{8} \oplus$, Elisabetta Garibaldi ${ }^{9}$, Adriana Faiella ${ }^{2}$,

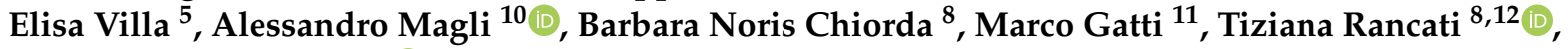 \\ Riccardo Valdagni ${ }^{8,12,13}{ }^{-}$, Nadia G. Di Muzio ${ }^{3}$, Claudio Fiorino ${ }^{3, *}$ and Cesare Cozzarini ${ }^{3}$
}

check for

updates

Citation: Munoz, F.; Sanguineti, G.;

Bresolin, A.; Cante, D.; Vavassori, V.; Waskiewicz, J.M.; Girelli, G.; Avuzzi, B.; Garibaldi, E.; Faiella, A.; et al. Predictors of Patient-Reported Incontinence at Adjuvant/Salvage Radiotherapy after Prostatectomy: Impact of Time between Surgery and Radiotherapy. Cancers 2021, 13, 3243. https://doi.org/10.3390/ cancers13133243

Academic Editors: Meritxell Arenas and Pierfrancesco Franco

Received: 7 May 2021

Accepted: 24 June 2021

Published: 29 June 2021

Publisher's Note: MDPI stays neutral with regard to jurisdictional claims in published maps and institutional affiliations.

Copyright: (c) 2021 by the authors. Licensee MDPI, Basel, Switzerland. This article is an open access article distributed under the terms and conditions of the Creative Commons Attribution (CC BY) license (https:// creativecommons.org/licenses/by/ $4.0 /)$.
1 SC Radioterapia Oncologica, Ospedale Regionale Parini-AUSL Valle d'Aosta, 11100 Aosta, Italy; fmunoz@ausl.vda.it

2 Deptartment of Radiotherapy, IRCCS Istituto Nazionale dei Tumori “Regina Elena”, 00144 Roma, Italy; giuseppe.sanguineti@ifo.gov.it (G.S.); adriana.faiella@ifo.gov.it (A.F.)

3 Deptartment of Medical Physics and Deptartment of Radiotherapy, IRCCS Istituto Scientifico Ospedale San Raffaele, 20132 Milano, Italy; breso88@gmail.com (A.B.); dimuzio.nadia@hsr.it (N.G.D.M.); cozzarini.cesare@hsr.it (C.C.)

4 Deptartment of Radiotherapy, ASL TO4, Ospedale di Ivrea, 10015 Ivrea, Italy; dcante@aslto4.piemonte.it

5 Deptartment of Radiotherapy, Cliniche Gavazzeni-Humanitas, 24125 Bergamo, Italy; vittorio.vavassori@gavazzeni.it (V.V.); elisa.villa@gavazzeni.it (E.V.)

6 Deptartment of Radiotherapy, Azienda Sanitaria dell'Alto Adige, 39100 Bolzano, Italy; justynamagdalena.waskiewicz@sabes.it

7 Deptartment of Radiotherapy, Ospedale degli Infermi, 22399 Biella, Italy; Giuseppe.Girelli@aslbi.piemonte.it

8 Deptartment of Radiation Oncology 1, Fondazione IRCCS Istituto Nazionale dei Tumori, 20133 Milan, Italy; Barbara.Avuzzi@istitutotumori.mi.it (B.A.); Barbara.Noris@istitutotumori.mi.it (B.N.C.); Tiziana.Rancati@istitutotumori.mi.it (T.R.); Riccardo.valdagni@istitutotumori.mi.it (R.V.)

9 SC Radioterapia, AO SS Antonio e Biagio e Cesare Arrigo Alessandria, 15121 Alessandria, Italy; elisabetta.garibaldi@ospedale.al.it

10 Deptartment of Radiotherapy, Azienda Ospedaliero Universitaria S. Maria della Misericordia, 33100 Udine, Italy; alessandro.magli@asufc.sanita.fvg.it

11 Istituto di Candiolo, Fondazione del Piemonte per l'Oncologia IRCCS, Candiolo, 10060 Torino, Italy; marco.gatti@ircc.it

12 Programma Prostata, Prostate Cancer Program, Fondazione IRCCS Istituto Nazionale dei Tumori, 20133 Milan, Italy

13 Deptartment of Oncology and Hemato-Oncology, Università degli Studi di Milano, 20122 Milan, Italy

* Correspondence: fiorino.claudio@hsr.it

Simple Summary: The levels of urinary incontinence (UI) at adjuvant/salvage radiotherapy (ART/ SRT) start strongly influence long-term UI recovery, possibly inducing clinicians to postpone radiotherapy "as much as possible" in order to maximize UI recovery, but possibly reducing radiotherapy efficacy. Our study analyzed UI recovery from prostatectomy to ART/SRT by means of the International Consultation on Incontinence Questionnaire-Short Form filled-in by patients at ART/SRT start. Three endpoints were investigated: frequency and amount of urine loss and the "subjective" patient-perceived detrimental impact on quality of life, as well as the possible influence of clinical and personality variables. The time elapsed from prostatectomy to radiotherapy start was the strongest predictor for each UI endpoint, all improving between four and eight months after prostatectomy, but without any additional long-term recovery.

Abstract: Background: Baseline urinary incontinence (UI) strongly modulates UI recovery after adjuvant/salvage radiotherapy (ART/SRT), inducing clinicians to postpone it "as much as possible", maximizing UI recovery but possibly reducing efficacy. This series aims to analyze the trend of UI recovery and its predictors at radiotherapy start. Methods: A population of 408 patients treated with ART/SRT enrolled in a cohort study (ClinicalTrials.gov \#NCT02803086) aimed at developing predictive models of radiation-induced toxicities. Self-reported UI and personality traits, evaluated 
by means of the International Consultation on Incontinence Questionnaire-Urinary Incontinence Short Form (ICIQ-SF) and Eysenck Personality Questionnaire - Revised (EPQ-R) questionnaires, were assessed at ART/SRT start. Several endpoints based on baseline ICIQ-SF were investigated: frequency and amount of urine loss (ICIQ3 and ICIQ4, respectively), “objective” UI (ICIQ3 + 4), "subjective" UI (ICIQ5), and "TOTAL" UI (ICIQ3 +4 + 5). The relationship between each endpoint and time from prostatectomy to radiotherapy (TTRT) was investigated. The association between clinical and personality variables and each endpoint was tested by uni- and multivariable logistic regression. Results: TTRT was the strongest predictor for all endpoints ( $p$-values $\leq 0.001$ ); all scores improved between 4 and 8 months after prostatectomy, without any additional long-term recovery. Neuroticism independently predicted subjective UI, TOTAL UI, and daily frequency. Conclusions: Early UI recovery mostly depends on TTRT with no further improvement after 8 months from prostatectomy. Higher levels of neuroticism may overestimate UI.

Keywords: prostatectomy; adjuvant radiotherapy; salvage radiotherapy; urinary incontinence; ICIQ-SF; EPQ-R

\section{Introduction}

Adjuvant radiotherapy (ART) was believed to reduce the risk of recurrence after radical prostatectomy $(\mathrm{RP})$ in patients with aggressive prostate cancer (PCa). However, three recently published non-inferiority phase III trials (RAVES, RADICALS, GETUG17), as well as the ARTISTIC meta-analysis, clearly indicated that salvage radiotherapy (SRT) at the time of recurrence may now be regarded as a preferred option in the large majority of patients [1-4].

Immediate ART could theoretically still be considered a valuable option in the presence of few pathological pejorative findings such as positive surgical margins and/or lymphnodal metastases: according to the ARTISTIC meta-analysis, owing to the low overall event rate, the power of the patient subgroup analyses indicating no superiority of ART when compared to early SRT, and also in the subset of men with positive surgical margins, was limited [1]. Similarly, the very low number of patients with positive lymph nodes enrolled in the three trials considered in the ARTISTIC meta-analysis prevented a thorough evaluation of the effect of radiotherapy timing in this patient subset.

A strong argument favoring SRT is the common belief that postponing post-prostatectomy irradiation until a better level of urinary incontinence (UI) has been achieved may result in a reduced risk of long-term radiotherapy-induced impairment of UI recovery [5]. This is indeed a crucial issue since the key role of UI with respect to both patient's preferences regarding the optimal management of newly diagnosed PCa [6] and long-term regret about the treatment received [7] is well recognized.

In 2016, Tendulkiar et al. elegantly highlighted an inverse relationship between preSRT baseline PSA and the risk of distant metastases and death following SRT, with men receiving SRT at PSA levels ranging from 0.01 to $0.20 \mathrm{ng} / \mathrm{mL}$ exhibiting better oncological outcome across all Gleason score subgroups [8], in agreement with the findings of a radiobiological model derived from a large multi-Institute database [9]. Nevertheless, even if a very early initiation of SRT may independently reduce the risk of subsequent disease progression $[2,3,8,10]$, some reports indicate that delaying post-prostatectomy radiotherapy (even in the case of ART) could exert a protective effect on long term UI recovery after RP [11-13]. Although this issue is still controversial [14-17], clinicians may increasingly have to deal with the dilemma of postponing SRT despite a rising PSA until satisfactory urinary continence is achieved, or proceed to immediate SRT at the first evidence of detectable PSA values regardless of an incompletely recovered pre-radiotherapy UI. In addition, it has been generally well ascertained that rigorous studies dealing with "patientreported" UI, which detail patient's quality of life better than "physician-reported" UI, 
are lacking [18]. It is equally acknowledged that baseline pre-radiotherapy UI is a strong predictor of long-term UI after radiotherapy [13,19].

Starting from these two assumptions, we sought to analyze the trend of UI recovery from RP to post-prostatectomy radiotherapy in a large cohort $(n=408)$ of patients treated with post-prostatectomy ART or SRT.

Special emphasis was placed on the possible identification of an optimal timing at which the start of postoperative radiotherapy could be regarded as adequately safe with respect to UI recovery.

The impact of clinical factors on baseline pre-radiotherapy UI and the possible influence of patient personality on the self-assessment of UI were also investigated.

\section{Patients and Methods}

\subsection{The IHU-WPRT TOX Study}

The multi-Institutional IHU-WPRT TOX study (https:/ / www.clinicaltrials.gov / (accessed on 1 June 2021) identifier \#NCT02803086) started in January 2014 with the goal of developing predictive models of hematological [20,21], patient-reported intestinal [22], and urinary toxicity from radiotherapy for PCa, including the prophylactic irradiation of the pelvic lymph-nodal area (whole pelvis radiotherapy; WPRT) for patients treated with ART, SRT, or with radical intent. The study was approved by the Institutional Review Board of both the coordinating Centre (San Raffaele Scientific Institute, Milan, protocol code $42 / 2014$, date of approval 6 February 2014) and of all the participating Institutes, and is still enrolling patients [20].

A single-institute pilot study, approved by the Institutional Review Board, was previously activated in October 2012 at the Coordinating Institute (San Raffaele Scientific Institute, Milan, protocol code 33/2012, date of approval 11 October 2012) [21,22]. Both studies were conducted according to the guidelines of the Declaration of Helsinki. Informed consent was obtained from all subjects involved in the two studies.

\subsection{The ICIQ-SF Questionnaire-Objective and Subjective UI}

According to the protocol requirements, the validated Italian version of the ICIQ-SF questionnaire (International Consultation on Incontinence Questionnaire-Short Form) [23] is to be filled in by the enrolled patients at baseline, at RT mid-point and end, 3 and 6 months after radiotherapy conclusion, and thereafter every 6 months up to 5 years. The ICIQ-SF comprises six items: questions 1 and 2 (dealing with personal data) and question 6 (descriptive) do not generate a score and were not considered. Questions 3 (hereafter ICIQ3, score 0-5) and 4 (ICIQ4, score 0-6) focus on the frequency and amount of urine loss, respectively.

The sum of the two scores, ICIQ3 + ICIQ4, pertains to the "objective" (OBJ) component of UI. Question 5 (ICIQ5, score 0-10) pertains to the subjective (SUBJ) patient-perceived impairment of quality of life attributable to UI. The total score (hereafter TOTAL, ICIQ3 + ICIQ4 + ICIQ5) ranging from 0 (best) to 21 (worst), therefore comprises both an "objective" and a "subjective" component. It is rather intuitive that QoL impairment deriving from similar frequency and amount of urine loss may be perceived differently by patients, and therefore all of the three distinct aspects of radiation-induced urinary incontinence, OBJ, SUBJ, and TOTAL will be separately analyzed in the present analysis.

\subsection{Role of Personality}

Patient personality and its impact on UI were considered by means of the abbreviated 24-item version of the revised Eysenck personality questionnaire (EPQ-R) [24] filled in by patients at baseline. Four personality traits, each scored from 0 to 6 , were evaluated: extraversion (sociability, impulsiveness, but also some tendency to aggressiveness), neuroticism (emotional instability, nervousness, and general anxiety), psychoticism (toughmindedness, but also a measurement of hostility), and lie (behaviors that are either socially desirable but infrequently practiced or frequently practiced but socially undesirable). 


\subsection{Patient Population}

This analysis pertains to the first 408 patients treated with ART ( $n=179,44 \%$ ) or SRT $(n=229,56 \%): 103$ were from the pilot study [20,21] and 305 from the observational protocol [22], enrolled in 11 Italian Institutes between 2011 and 2019 and for whom a complete ICIQ-SF at baseline was available. See Table 1 for patient characteristics.

Table 1. Summary of patient characteristics. Data are presented as counts (percentages in brackets) for categorical variables and as median values (interquartile range in bracket) for continuous variables.

\begin{tabular}{|c|c|c|}
\hline Age (years) & 67 & $(62-71)$ \\
\hline $\mathrm{BMI}\left(\mathrm{kg} / \mathrm{m}^{2}\right)$ & 26 & $(24-28)$ \\
\hline Time to RT (mo) & 7.7 & $(4.1-26.4)$ \\
\hline \multicolumn{3}{|l|}{ PSA (ng/mL) } \\
\hline pre-RP & 8.58 & $(5.83-13.98)$ \\
\hline post-RP & 0.04 & $(0.01-0.15)$ \\
\hline pre-RT & 0.22 & $(0.05-0.48)$ \\
\hline Number of removed lymph nodes & 13 & $(7-21)$ \\
\hline Hypertension & 178 & $(44 \%)$ \\
\hline Smoke & 69 & $(18 \%)$ \\
\hline Diabetes & 27 & $(7 \%)$ \\
\hline \multicolumn{3}{|l|}{ ADT } \\
\hline No ADT & 213 & $(58 \%)$ \\
\hline Bicalutamide monotherapy & 33 & $(9 \%)$ \\
\hline LH-RH & 89 & $(24 \%)$ \\
\hline $\mathrm{CAB}$ & 30 & $(8 \%)$ \\
\hline \multicolumn{3}{|l|}{ Surgical margins } \\
\hline Negative & 237 & $(58 \%)$ \\
\hline Positive & 171 & $(42 \%)$ \\
\hline \multicolumn{3}{|l|}{ Surgery } \\
\hline Open & 233 & $(59 \%)$ \\
\hline Robotic & 120 & $(30 \%)$ \\
\hline Laparoscopic & 43 & $(11 \%)$ \\
\hline \multicolumn{3}{|l|}{ Gleason score } \\
\hline ISUP Groups 1-3 & 102 & $(27 \%)$ \\
\hline ISUP Groups 4-5 & 274 & $(73 \%)$ \\
\hline \multicolumn{3}{|l|}{ Stage $\mathrm{T}$} \\
\hline pT2 & 121 & $(30 \%)$ \\
\hline pT3a & 143 & $(35 \%)$ \\
\hline pT3b and pT4 & 139 & $(34 \%)$ \\
\hline
\end{tabular}

$\overline{\mathrm{BMI}}$ = body mass index; $\mathrm{RP}$ = radical prostatectomy; $\mathrm{RT}$ = radiotherapy; $\mathrm{ADT}$ = androgen deprivation therapy; $\mathrm{CAB}=$ combined androgen blockade.

With respect to the indication of WPRT, provided that it was at the discretion of the referring radiation oncologist of every participating Institute, it was usually advised for patients with seminal vesicle invasion, Gleason score $\geq 7$, pre-surgical PSA $>10 \mathrm{ng} / \mathrm{mL}$ and/or histologically positive lymph-nodes at prostatectomy, or in the case of a PSA $\geq 0.50 \mathrm{ng} / \mathrm{mL}$ in the salvage setting. 


\subsection{ICIQ Based Incontinence Endpoints}

The ICIQ based endpoints were selected following, as a reference, a previous analysis on UI in the radical setting [19] and evaluating the distribution of the ICIQ scores (Table S1) in order to establish clinically relevant criteria. In summary, the following five endpoints scored at baseline were considered:

(a) daily frequency: ICIQ3 $>2$

(b) amount of urine loss: ICIQ4 $>2$

(c) subjective: ICIQ5 $>4$

(d) objective: ICIQ3 + ICIQ4 $>5$

(e) total: ICIQ3 + ICIQ4 + ICIQ5 > 8

\subsection{Statistical Methods}

Relationship between ICIQ Scores and Time from Prostatectomy

First, the relationship between UI at radiotherapy and the time elapsed between prostatectomy and radiotherapy (time to radiotherapy, TTRT) was investigated. The scatter plot of each ICIQ-based score versus TTRT was represented. Data were grouped into deciles with respect to the distribution along the TTRT axis: mean values and their standard errors were considered to be representative of each decile interval. Points were fitted by a sigmoid curve with initial parameters manually selected and then optimized by the $n l s$ function implemented in the $\mathrm{R}$ package stats. Coefficient estimation was based on an iterative process, which entails a linearization approximation leading to a least-squares problem at each step.

Subsequently, the best cut-off values for the TTRT, which best discriminate the highest risk of experiencing each of the five previously defined endpoints were identified by means of ROC curve analyses.

\subsection{Logistic Regression Analysis and Internal Validation}

The possible correlation between clinical and EPQ-R variables (see Table 2) with each endpoint tested by univariable logistic analysis.

Table 2. Odds ratios (95\% CI in brackets) and $p$-values resulting from univariable logistic regression analyses: correlation with the ICIQ based endpoints. $p$-values $<0.1$ are in bold.

\begin{tabular}{|c|c|c|c|c|c|c|}
\hline \multicolumn{2}{|c|}{ Parameter } & $\begin{array}{c}\text { Daily } \\
\text { Frequency }\end{array}$ & $\begin{array}{l}\text { Amount of } \\
\text { Urine Loss }\end{array}$ & Subjective & Objective & Total \\
\hline \multirow{2}{*}{\multicolumn{2}{|c|}{ Age (yr) }} & $1.03(1.00-1.06)$ & $1.00(0.96-1.05)$ & $1.01(0.97-1.04)$ & 1.03 (1.00-1.06) & $1.02(0.99-1.05)$ \\
\hline & & 0.062 & 0.812 & 0.697 & 0.080 & 0.189 \\
\hline \multirow{2}{*}{\multicolumn{2}{|c|}{ BMI $\left(\mathrm{kg} / \mathrm{m}^{2}\right)$}} & $1.05(0.99-1.11)$ & $1.05(0.97-1.13)$ & $1.06(0.99-1.13)$ & $1.04(0.98-1.10)$ & $1.06(0.99-1.12)$ \\
\hline & & 0.091 & 0.218 & 0.112 & 0.230 & 0.082 \\
\hline \multirow{6}{*}{ PSA (ng/mL) } & \multirow{2}{*}{ pre-RP } & $1.02(1.00-1.04)$ & $1.01(0.99-1.03)$ & $1.01(1.00-1.03)$ & $1.01(1.00-1.03)$ & $1.01(1.00-1.03)$ \\
\hline & & 0.024 & 0.134 & 0.104 & 0.032 & 0.063 \\
\hline & \multirow{2}{*}{ post-RP } & $0.97(0.77-1.19)$ & $1.14(0.88-1.41)$ & $1.00(0.73-1.25)$ & $1.00(0.78-1.22)$ & $0.99(0.76-1.22)$ \\
\hline & & 0.775 & 0.250 & 0.981 & 0.971 & 0.922 \\
\hline & \multirow{2}{*}{ pre-RT } & $0.98(0.90-1.05)$ & $1.04(0.96-1.11)$ & $1.02(0.94-1.09)$ & $1.00(0.93-1.07)$ & 0.99 (0.91-1.06) \\
\hline & & 0.587 & 0.303 & 0.518 & 0.977 & 0.846 \\
\hline \multicolumn{7}{|c|}{ Time to RT (mo) } \\
\hline \multicolumn{2}{|c|}{$\geq$ cut-off time } & Ref. & Ref. & Ref. & Ref. & Ref. \\
\hline \multirow{2}{*}{\multicolumn{2}{|c|}{$<$ cut-off time }} & $3.30(2.20-5.00)$ & $3.55(1.94-6.81)$ & $2.31(1.41-3.86)$ & $2.94(1.92-4.57)$ & $3.16(2.02-5.01)$ \\
\hline & & $<0.0001$ & $<0.0001$ & 0.001 & $<0.0001$ & $<0.0001$ \\
\hline
\end{tabular}


Table 2. Cont.

\begin{tabular}{|c|c|c|c|c|c|c|}
\hline \multicolumn{2}{|c|}{ Parameter } & $\begin{array}{c}\text { Daily } \\
\text { Frequency }\end{array}$ & $\begin{array}{l}\text { Amount of } \\
\text { Urine Loss }\end{array}$ & Subjective & Objective & Total \\
\hline \multirow{2}{*}{\multicolumn{2}{|c|}{$\mathrm{N}^{\circ}$ removed lymph nodes }} & $1.01(0.99-1.03)$ & $1.02(0.99-1.04)$ & $1.01(0.99-1.03)$ & $1.01(0.99-1.03)$ & $1.01(0.99-1.03)$ \\
\hline & & 0.368 & 0.113 & 0.275 & 0.549 & 0.424 \\
\hline \multirow[t]{3}{*}{ Hypertension } & No & Ref. & Ref. & Ref. & Ref. & Ref. \\
\hline & & $1.16(0.78-1.73)$ & $1.00(0.57-1.74)$ & $1.09(0.67-1.77)$ & $1.16(0.76-1.76)$ & $0.99(0.64-1.51)$ \\
\hline & Yes & 0.460 & 1.000 & 0.722 & 0.495 & 0.945 \\
\hline \multirow[t]{3}{*}{ Smoke } & No & Ref. & Ref. & Ref. & Ref. & Ref. \\
\hline & Yes & $1.05(0.62-1.77)$ & $1.60(0.80-3.07)$ & $1.30(0.69-2.37)$ & $1.33(0.77-2.28)$ & $1.29(0.73-2.22)$ \\
\hline & res & 0.864 & 0.165 & 0.399 & 0.300 & 0.372 \\
\hline \multirow[t]{3}{*}{ Diabetes } & No & Ref. & Ref. & Ref. & Ref. & Ref. \\
\hline & & $2.46(1.12-5.71)$ & $1.36(0.44-3.48)$ & $0.46(0.11-1.37)$ & $1.51(0.66-3.32)$ & $1.41(0.61-3.13)$ \\
\hline & Yes & 0.029 & 0.553 & 0.219 & 0.313 & 0.407 \\
\hline \multirow[t]{3}{*}{ ADT } & No & Ref. & Ref. & Ref. & Ref. & Ref. \\
\hline & Yes & $1.54(1.04-2.31)$ & $1.84(1.05-3.25)$ & $1.31(0.81-2.13)$ & $1.52(1.00-2.32)$ & 1.67 (1.09-2.58) \\
\hline & res & 0.033 & 0.034 & 0.274 & 0.052 & 0.019 \\
\hline \multirow[t]{5}{*}{ Surgery } & Open & Ref. & Ref. & Ref. & Ref. & Ref. \\
\hline & & $0.87(0.55-1.36)$ & $1.18(0.63-2.17)$ & $0.94(0.54-1.61)$ & $0.92(0.57-1.48)$ & $0.83(0.50-1.35)$ \\
\hline & Robotic & 0.547 & 0.595 & 0.821 & 0.737 & 0.456 \\
\hline & & $1.07(0.55-2.06)$ & $1.44(0.58-3.25)$ & $0.99(0.42-2.13)$ & $1.27(0.64-2.48)$ & $1.22(0.60-2.40)$ \\
\hline & Laparoscopic & 0.836 & 0.406 & 0.988 & 0.483 & 0.566 \\
\hline \multicolumn{7}{|c|}{ Surgical margins } \\
\hline & Negative & Ref. & Ref. & Ref. & Ref. & Ref. \\
\hline \multirow{2}{*}{\multicolumn{2}{|c|}{ Positive }} & $1.23(0.82-1.86)$ & $1.12(0.64-1.99)$ & $1.06(0.65-1.75)$ & $1.12(0.73-1.72)$ & $1.03(0.67-1.59)$ \\
\hline & & 0.313 & 0.696 & 0.803 & 0.609 & 0.903 \\
\hline \multicolumn{7}{|c|}{ Gleason score } \\
\hline \multicolumn{2}{|c|}{ ISUP Groups 1-3 } & Ref. & Ref. & Ref. & Ref. & Ref. \\
\hline \multirow{2}{*}{\multicolumn{2}{|c|}{ ISUP Groups 4-5 }} & $1.49(0.94-2.41)$ & $1.67(0.86-3.52)$ & $115(0.66-2.06)$ & $1.5(0.91-2.51)$ & $1.34(0.81-2.25)$ \\
\hline & & 0.095 & 0.153 & 0.631 & 0.117 & 0.263 \\
\hline \multirow[t]{5}{*}{ Stage $\mathrm{T}$} & pT2 & Ref. & Ref. & Ref. & Ref. & Ref. \\
\hline & & $1.50(0.90-2.52)$ & $1.53(0.71-3.46)$ & $1.00(0.53-1.89)$ & $2.06(1.19-3.65)$ & $1.4(0.8-2.47)$ \\
\hline & p13a & 0.117 & 0.287 & 1.000 & 0.011 & 0.240 \\
\hline & pT $3 b$ and pT4 & $2.58(1.56-4.34)$ & $2.64(1.29-5.76)$ & $1.51(0.84-2.79)$ & $2.44(1.41-4.31)$ & $2.15(1.25-3.75)$ \\
\hline & prov ancu pit & $<0.001$ & 0.011 & 0.175 & 0.002 & 0.006 \\
\hline \multicolumn{7}{|c|}{ EPQ-R } \\
\hline \multirow{2}{*}{\multicolumn{2}{|c|}{ Extroversion }} & $0.97(0.85-1.10)$ & $0.90(0.75-1.06)$ & $0.96(0.82-1.12)$ & $0.93(0.81-1.06)$ & $0.90(0.79-1.03)$ \\
\hline & & 0.617 & 0.202 & 0.555 & 0.270 & 0.134 \\
\hline \multirow{2}{*}{\multicolumn{2}{|c|}{ Neuroticism }} & 1.18 (1.04-1.35) & $1.16(0.97-1.38)$ & $1.22(1.04-1.42)$ & 1.15 (1.00-1.32) & $1.19(1.04-1.37)$ \\
\hline & & 0.012 & 0.098 & 0.012 & 0.049 & 0.014 \\
\hline \multirow{2}{*}{\multicolumn{2}{|c|}{ Psychoticism }} & $0.94(0.77-1.14)$ & $1.07(0.82-1.38)$ & $1.04(0.82-1.30)$ & $1.00(0.81-1.22)$ & $0.97(0.78-1.18)$ \\
\hline & & 0.518 & 0.596 & 0.754 & 0.992 & 0.741 \\
\hline \multirow{2}{*}{\multicolumn{2}{|c|}{ Lie }} & $1.00(0.85-1.19)$ & $0.93(0.75-1.17)$ & 1.07 (0.87-1.33) & $1.06(0.89-1.28)$ & $1.06(0.89-1.28)$ \\
\hline & & 0.981 & 0.511 & 0.557 & 0.533 & 0.523 \\
\hline
\end{tabular}

ICIQ3, ICIQ4, ICIQ5 = score of the International Consultation on Incontinence Modular Questionnaire Short Form for questions three, four and five respectively; $\mathrm{BMI}=$ body mass index; $\mathrm{RP}$ = radical prostatectomy; $\mathrm{RT}=$ radiotherapy; $\mathrm{ADT}=$ androgen deprivation therapy; $\mathrm{EPQR}$ $=$ Eysenck Personality Questionnaire-Revised. 
Mean imputation was performed for EPQ-R variables when only one answer for each personality trait was missing $(n=23)$. For 46 patients, the EPQ-R questionnaire was not available owing to patient refusal or largely incomplete forms.

Variables with a $p$-value $<0.1$ at univariable analyses were entered into a backward stepwise multivariable (MVA) logistic regression model. Each model was then reprocessed using only the variables retained by the MVA model with a $p$-value threshold $\leq 0.1$. The multivariable analyses were subsequently repeated, excluding the personality variables.

Goodness of fit was assessed by means of the Hosmer and Lemeshow test (H\&L) and the calibration of the models evaluated through the calibration plot (slope and regression coefficient $\mathrm{R}^{2}$ ). Internal validation was performed by 1000 bootstrap resampling: the Brier scores after bootstrapping were considered with and without optimism taken into account. Analyses were performed with MedCalc ${ }^{\circledR}$ version 12.1.4.0 (MedCalc Software, Mariakerke Brussels, Belgium) and the R software version 3.2.4 (@The R Foundation for Statistical Computing, Vienna, Austria).

\section{8. "Completely Dry" Patients}

The predictors of the "completely-dry" condition (ICIQ3 + ICIQ4 $=0,27 \%$ of the population) at post-prostatectomy radiotherapy start were analyzed with the same procedure followed for the identification of the predictors of the five above described UI endpoints.

\section{Results}

\subsection{Relationship between Baseline ICIQ Scores and TTRT}

The relationships between TTRT and the different ICIQ scores are plotted in Figure 1.

All figures show an initial post-surgical plateau of the different UI aspects followed by a partial recovery and then by a new and definitive plateau. The most informative cut-offs in terms of TTRT with respect to the five previously defined UI endpoints ranged from 6.7 to 7.8 months. The coefficients of the sigmoid curves, the corresponding $p$-values, and the best cut-off values are listed in Table S2.

\subsection{Predictors of Pre-Radiotherapy UI}

The results of the univariate logistic regression are shown in Table 2. Not unexpectedly, TTRT emerged as the strongest predictor with respect to all the considered endpoints ( $p$-value always $\leq 0.001)$. With respect to the clinical variables, the $p$-values for androgen deprivation therapy, pathologic T-stage, and pre-RP PSA were always $<0.1$ with the sole exception of the SUBJ endpoint, while, with respect to the role of patient personality, neuroticism always emerged as a significant factor, unlike the other three personality traits. Of note, no correlation between the type of surgery and any of the considered endpoints was observed ( $p$-value always $>0.2$ ).

The resulting multivariate models, shown in Table 3, confirmed TTRT as the strongest predictor of UI, with odds ratios (ORs) ranging from 2.55 for SUBJ to 3.57 for daily frequency (ICIQ3), and resulting as the only independent predictor for the amount of urine loss (ICIQ4) and OBJ endpoints. 

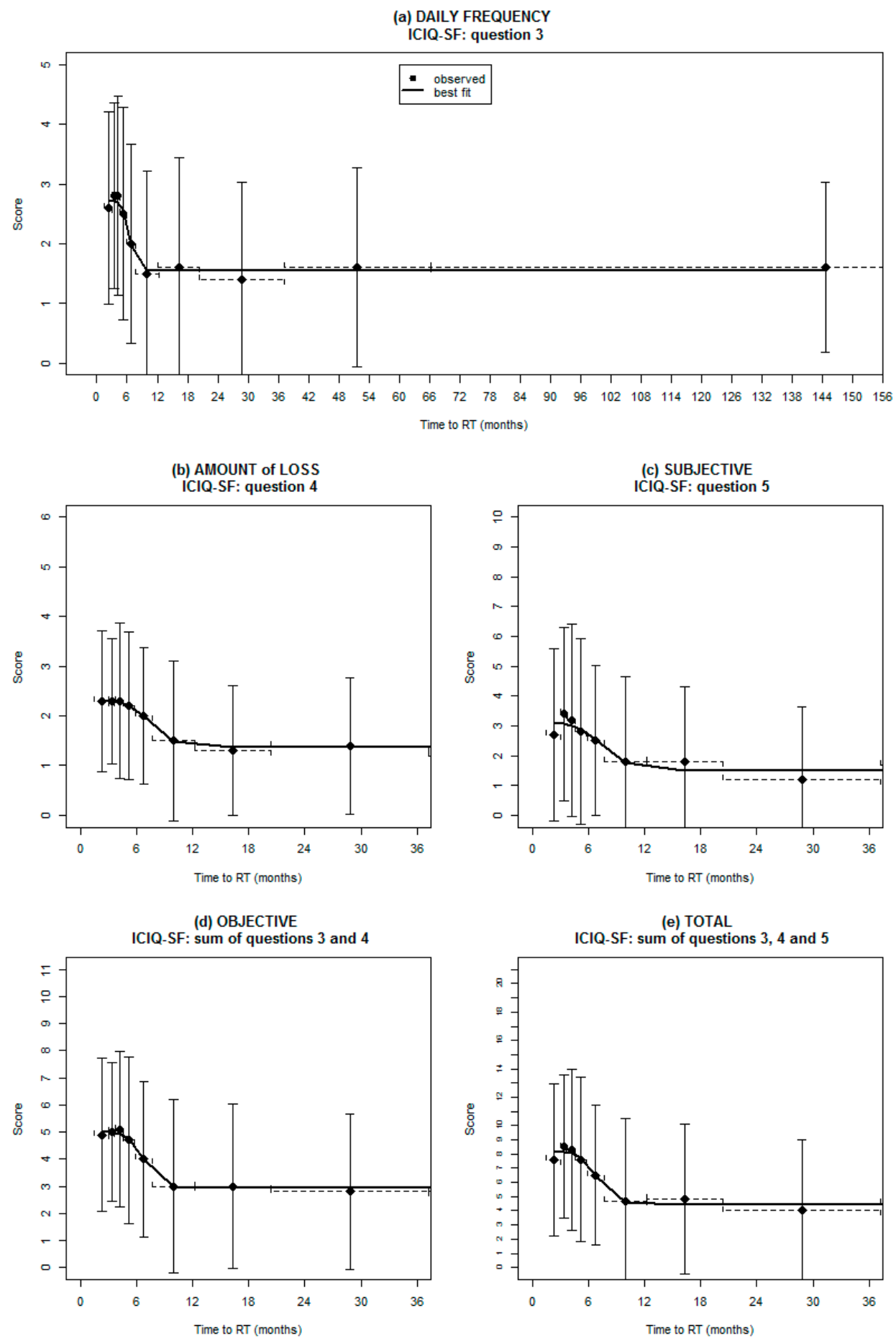

Figure 1. A plot of the relationship between incontinence symptoms and time elapsed between prostatectomy and post-prostatectomy radiotherapy. Incontinence was evaluated through the International Consultation on Incontinence Questionnaire Short Form (ICIQ-SF). Points represent the mean values of the ICIQ score in each decile interval; bars show the standard deviations and continuous lines and the fit between the data and a sigmoid curve. Each plot is associated with the frequency (a) and amount (b) of urine leakage, the subjective perceived quality of life (c), the objective (d), and total (e) estimation of urinary incontinence, respectively. The time axis for endpoint (a) covered the last decile to show the entire plateau (the same behavior was found for all the remaining endpoints). For the remaining endpoints, the scale was restricted to the first 3-years to render the results more easily comprehensible. 
Table 3. Results of backward stepwise multivariable logistic regression analyses performed with the most significant variables $(p<0.1)$. The main performances of the models are also reported.

\begin{tabular}{|c|c|c|c|}
\hline End-Po Int & Coeff. \pm St. dev. & Odds Radio (95\% CI) & $p$-Value \\
\hline \multicolumn{4}{|c|}{ (a) Daily Frequency endpoint: ICIQ3 > 2 ( $n=152 / 372,41 \%)$} \\
\hline TTRT (cut-off: $6.7 \mathrm{mo}$ ) & $1.272 \pm 0.223$ & $3.57(2.31-5.56)$ & $<0.0001$ \\
\hline Neuroticism & $0.167 \pm 0.070$ & $1.18(1.03-1.36)$ & 0.018 \\
\hline Constant: & -1.266 & & \\
\hline \multicolumn{4}{|c|}{$\begin{array}{c}\text { H\&L } p \text {-value }=0.693 ; \text { Brier score }=0.216 \text { (corrected for optimism: } 0.219) \text {; calibration slope }=0.975 ; R^{2}=0.840 \\
\text { (b) Amount of Loss endpoint: ICIQ4 }>2(n=59 / 408, \mathbf{1 4} \%)\end{array}$} \\
\hline TTRT (cut-off: $7.2 \mathrm{mo}$ ) & $1.266 \pm 0.318$ & $3.55(1.94-6.81)$ & 0.0001 \\
\hline Constant: & -2.544 & & \\
\hline \multicolumn{4}{|c|}{$\begin{array}{c}\text { H\&L } p \text {-value }=1.000 ; \text { Brier score }=0.118(\text { corrected for optimism: } 0.120) ; \text { calibration slope }=1.000 ; R^{2}=1.000 \\
\text { (c) Subjective endpoint: ICIQ5 }>4(n=75 / 372,20 \%)\end{array}$} \\
\hline TTRT (cut-off: $7.1 \mathrm{mo}$ ) & $0.935 \pm 0.272$ & $2.55(1.51-4.39)$ & 0.001 \\
\hline Neuroticism & $0.190 \pm 0.080$ & $1.21(1.03-1.42)$ & 0.018 \\
\hline Constant: & -2.241 & & \\
\hline \multicolumn{4}{|c|}{ 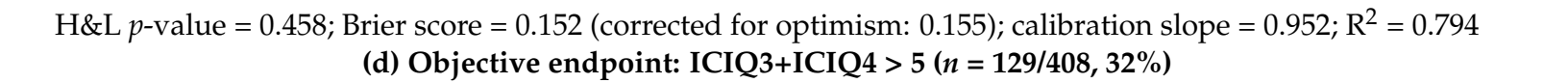 } \\
\hline TTRT (cut-off: $6.7 \mathrm{mo}$ ) & $1.080 \pm 0.221$ & $2.94(1.92-4.57)$ & $<0.001$ \\
\hline Constant: & -1.325 & & \\
\hline \multicolumn{4}{|c|}{$\begin{array}{c}\text { H\&L } p \text {-value }=1.000 ; \text { Brier score }=0.203(\text { corrected for optimism: } 0.205) \text {; calibration slope }=1.000 ; R^{2}=1.000 \\
\text { (e) Total endpoint: ICIQ3+ICIQ4+ICIQ5 }>\mathbf{8}(\boldsymbol{n}=\mathbf{1 0 8 / 3 6 5 , 3 0 \% )}\end{array}$} \\
\hline TTRT (cut-off: $7.8 \mathrm{mo}$ ) & $1.206 \pm 0.246$ & $3.34(2.08-5.46)$ & $<0.0001$ \\
\hline Neuroticism & $0.155 \pm 0.075$ & $1.17(1.01-1.35)$ & 0.039 \\
\hline BMI & $0.064 \pm 0.034$ & $1.07(1.00-1.14)$ & 0.058 \\
\hline Constant: & -2.569 & & \\
\hline \multicolumn{4}{|c|}{ H\&L $p$-value $=0.454 ;$ Brier score $=0.188($ corrected for optimism: 0.193$) ;$ calibration slope $=1.112 ; R^{2}=0.633$} \\
\hline & Coeff. \pm St. dev. & Odds radio $(95 \% \mathrm{CI})$ & $p$-value \\
\hline \multicolumn{4}{|c|}{ Completely Dry endpoint: ICIQ3+ICIQ4 = $0(n=108 / 408,26 \%)$} \\
\hline TTRT (cut-off: $6.9 \mathrm{mo}$ ) & $-1.429 \pm 0.257$ & $0.24(0.14-0.40)$ & $<0.0001$ \\
\hline Age & $-0.059 \pm 0.017$ & $0.94(0.90-0.98)$ & 0.001 \\
\hline Constant: & 3.445 & & \\
\hline \multicolumn{4}{|c|}{ H\&L $p$-value $=0.185 ;$ Brier score $=0.176($ corrected for optimism: 0.178$) ;$ calibration slope $=0.956 ; R^{2}=0.837$} \\
\hline
\end{tabular}

EPQ-R = Eysenck Personality Questionnaire Revised; ICIQ-SF = International Consultation on Incontinence Modular Questionnaire Short Form; TTRT = time-to-radiotherapy; H\&L = Hosmer and Lemeshow test.

Interestingly, neuroticism was confirmed as an independent predictor of SUBJ and TOTAL UI as well as, quite unexpectedly, of daily frequency.

The goodness of fits were always satisfactory (H\&L $>0.05$ and Brier score $<0.216$ ). For the models including more than one variable, the calibration showed excellent performance, with slopes ranging from 0.95 to 1.11 , and with $\mathrm{R}^{2}$ ranging from 0.63 to 0.84 . All calibration plots are shown in Figure S1. The bootstrap-based internal validation confirmed the robustness of the results: the Brier scores corrected for optimism changed by only 0.002 0.004 relative to the values of the original model.

The corresponding models obtained excluding the EPQ-R variables are shown in Table S3 and Figure S2. When disregarding the possible influence of patient personality, the independent role of age in increasing the risk of both daily frequency and OBJ (OR 1.03 and 1.04, respectively) emerged (Table S3).

\subsection{Predictors of the "Completely-Dry" Condition}

A summary of the results of univariate and multivariate analyses are shown in Table S4 and Table 3, respectively. The two most significant predictors, confirmed by multivariate analysis, were age and TTRT, indicating a higher probability of being "completely-dry" at post-prostatectomy radiotherapy for younger ( $\leq 69$ years) patients starting radiotherapy $\geq 7$ months after surgery. The resulting two-variable model showed an excellent calibration (slope $=0.956, \mathrm{R}^{2}=0.837$ ). These results were confirmed even after having excluded the role of personality (Table S3). In Figure S3 the fraction of "completely dry" patients against 
the time between prostatectomy and radiotherapy was shown according to patient age ( $\leq 69$ years vs. $>69$ years). In Figure 2, the relationship between the rate of "completely-dry" patients and TTRT is plotted: the rate rapidly increased between four and ten months after surgery from about 10 to $40 \%$, with no further improvements for longer periods after surgery.

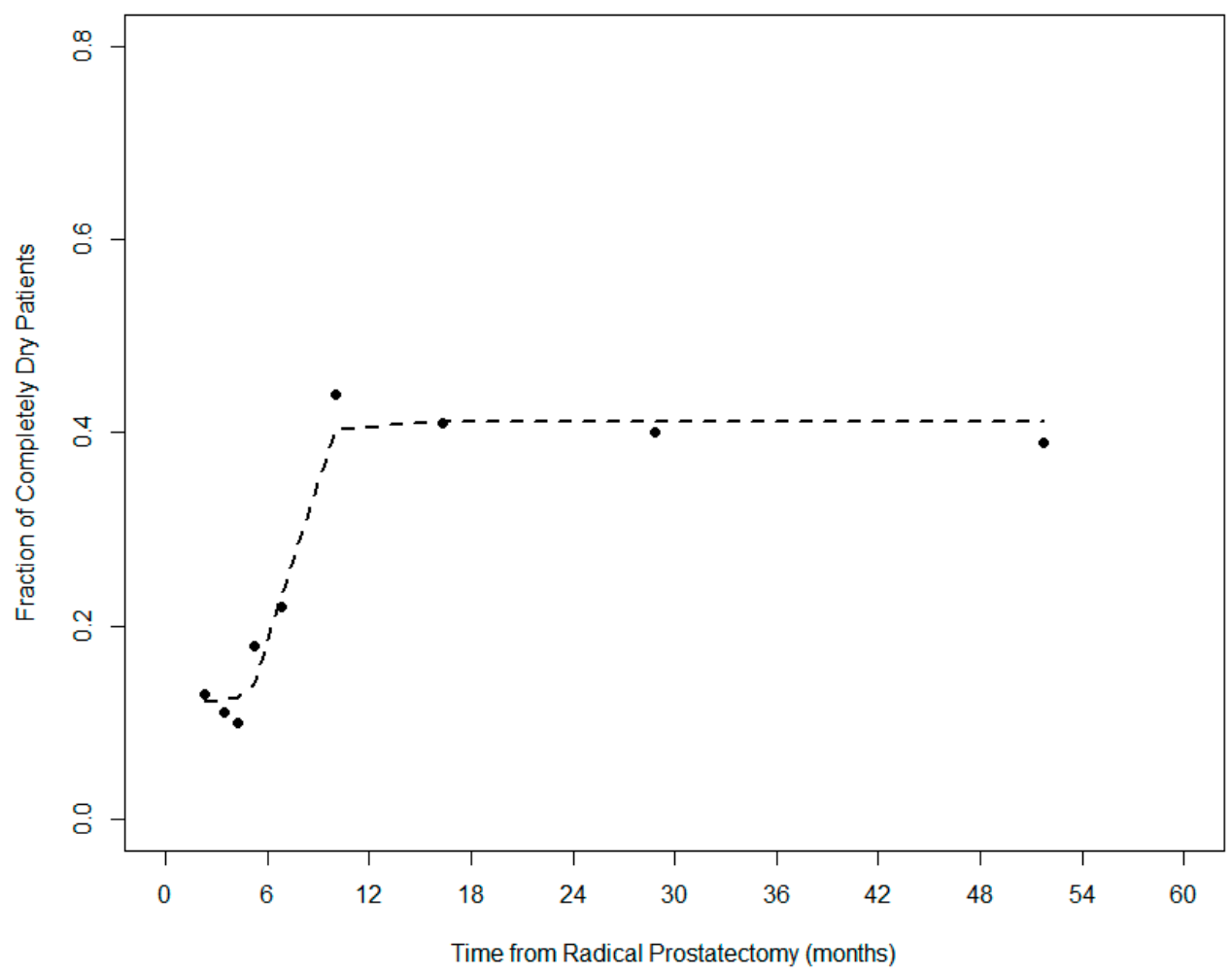

Figure 2. A plot of the fraction of "completely dry" patients (showing ICIQ3 + ICIQ4 = 0) against the time between prostatectomy and radiotherapy. The rate increased sharply between 4 and 10 months after surgery, reaching a plateau value of around $40 \%$ even at much longer periods.

\section{Discussion}

To the best of our knowledge, this is the first analysis of a large cohort of patients treated with post-prostatectomy radiotherapy focused on both the time-dependence of patient-reported UI recovery after radical prostatectomy and the identification of clinical and personality-related variables independently predictive of baseline pre-radiotherapy UI in its various and complex facets.

The first (and relatively unexpected) finding was that no further significant improvement of pre-radiotherapy baseline UI was recorded beyond 7-8 months after prostatectomy.

As pre-radiotherapy baseline UI was expected to be the strongest predictor of postirradiation late UI $[19,25,26]$, this finding heavily undermines the common propensity to postpone the beginning of radiotherapy for as long as possible in order to improve urinary outcome after ART or SRT [11]. Of note, some patients are candidates to receive ADT in combination with both ART and SRT, and for them, the postponement of irradiation is therefore of less importance. According to our findings, all the extremely multifaceted aspects of UI showed a similar relationship with TTRT, with an initial quasi-plateau up to 3-4 months from prostatectomy characterized by the highest (worst) ICIQ mean scores, followed by a rapid recovery in the subsequent $3-4$ months and by a second plateau with much lower (though significantly greater than zero) ICIQ scores, but with no evidence of further recovery, even years after prostatectomy. Based on this result, the optimal "compromise" between the earliest possible postoperative irradiation and the best UI recovery falls within the range of $7-8$ months after surgery, which should result in a 
2.5-3.5 fold reduction of the probability of starting radiotherapy with moderate to severe UI still present. As baseline UI is expected to impact post-radiotherapy UI recovery, our results are consistent with those of van Stam and coworkers, who observed that patients starting SRT seven months or more after RP were more likely to recover urinary function after irradiation [13].

If further confirmed, these findings could lead to a dramatic reduction in the current, apparently unbridgeable, gulf between ART and early SRT.

No significant differences in terms of UI were observed among patients managed with different surgical approaches, as already noted [27-29]. Nevertheless, our findings should be interpreted with extreme caution, owing to the possibly heterogeneous skill levels of surgeons operating in the Institutes where the study was conducted.

Interestingly, one-fourth of the patients included in this analysis were completely dry at the start of post-prostatectomy radiotherapy, representing undeniable proof of the significant improvement in the surgical management of prostate cancer. The complete recovery from UI before post-prostatectomy radiotherapy was, as expected, more frequent in younger patients and strongly related to TTRT (>7 months). Nevertheless, the rate of the completely dry patients (which significantly increased up to $40 \%$ in patients who started radiotherapy ten months after surgery), permanently plateaued, even when several years had elapsed from surgery; clearly indicating that waiting more than ten months after prostatectomy does not further increase the likelihood of starting radiotherapy in a "completely dry" condition.

As a final remark, higher levels of neuroticism, i.e., emotional instability, nervousness, and general anxiety, were associated with a reduced capacity to cope with UI, leading to an increased perception of impaired QoL deriving from UI, but also to a possible overestimation of daily frequency (ICIQ3 score $>2$, OR 1.18, Table 3).

Excluding the personality-related variables, an independent role of age in increasing the risk of UI (Table S3), as already occasionally reported [13,30], has emerged. This role appeared to be even more notable when focusing on the subset of "completely dry" patients, with an OR of 0.94/year.

An important limitation of the current analysis, based on a prospective study originally aimed at investigating the predictors of acute and late radiation-induced toxicities, is the lack of any pre-surgical assessment of urinary functionality as well as the lack of information regarding perineal re-education and medications for urinary frequency after surgery.

\section{Conclusions}

Both objective and subjective UI improved notably in the first 7-8 months after RP in candidates for post-prostatectomy radiotherapy, but with no further significant recovery for longer periods after surgery.

Higher levels of neuroticism may lead to some overestimation of UI.

Supplementary Materials: The following are available online at https: / www.mdpi.com/article/10 .3390 / cancers13133243/s1, Figure S1: Calibration plot of the final multivariate model related to each ICIQ based endpoint, Figure S2: Calibration plot of the final multivariate model related to each ICIQ based endpoint. EPQ-R variables were excluded from these analyses, Figure S3: Plot of the fraction of "completely dry" patients (showing ICIQ3 + ICIQ4 = 0) against the time between prostatectomy and radiotherapy, according to patient age ( $\leq 69$ years vs. $>69$ years), Table S1: Median value and interquartile range of scores in the EPQ-R and in ICIQ-SF questionnaires at baseline (immediately before the beginning of the radiotherapy), Table S2: Coefficients, standard deviation, and $p$-value of the sigmoid curve fitting. The most informative cut-off, which discriminates each endpoint in terms of time-to-RT, is also reported, Table S3: Results of backward stepwise multivariable logistic regression analyses performed with the most significant variables $(p<0.1)$ excluding EPQ-R predictors. The main performances of the models are also reported, Table S4: $p$-values and odds ratios (95\% CI in bracket) resulting from univariable logistic regression analyses related to the "completely dry" endpoint. $p$-values $<0.1$ are in bold. 
Author Contributions: Conceptualization, F.M., A.B., C.F., and C.C.; methodology, A.B. and C.F.; software, A.B. and C.F.; validation, F.M., G.S., A.B., C.F., and C.C.; formal analysis, A.B. and C.F.; investigation, F.M., G.S., and C.C.; resources, F.M., G.S., D.C., V.V., J.M.W., G.G., B.A., E.G., and A.F., E.V., A.M., B.N.C., M.G., N.G.D.M., C.C.; data curation, A.B., C.F., and C.C.; writing-original draft preparation, F.M., G.S., A.B., C.F., and C.C.; writing-review and editing, F.M., G.S., A.B., D.C., V.V., J.M.W., G.G., B.A., E.G., A.F., E.V., A.M., B.N.C., M.G., T.R., R.V., N.G.D.M., C.F., and C.C.; visualization, C.F. and C.C.; supervision, F.M., G.S., C.F., and C.C.; project administration, C.C.; funding acquisition, C.C. All authors have read and agreed to the published version of the manuscript.

Funding: The research leading to these results has received funding from AIRC (Associazione Italiana Ricerca Cancro) under Investigational Grant 2014-ID. 14603 project_-Principal Investigator Cesare Cozzarini.

Institutional Review Board Statement: The study was approved by the Institutional Review Board of both the coordinating Centre (San Raffaele Scientific Institute, protocol code 42/2014, date of approval 6 February 2014) and of all the participating Institutes. A single-institute pilot study, approved by the Institutional Review Board, was previously activated in October 2012 at the Coordinating Institute (San Raffaele Scientific Institute, Milan, protocol code 33/2012, date of approval 11 October 2012) $[21,22]$. Both studies were conducted according to the guidelines of the Declaration of Helsinki.

Informed Consent Statement: Informed consent was obtained from all subjects involved in the two studies.

Data Availability Statement: All original data will be made available upon request.

Acknowledgments: Cinzia Bianconi and Fabio Badenchini are acknowledged for data-managing.

Conflicts of Interest: The authors declare no conflict of interest. The funders had no role in the design of the study, in the collection, analyses, or interpretation of data, in the writing of the manuscript, or in the decision to publish the results.

\section{References}

1. Vale, C.L.; Fisher, D.; Kneebone, A.; Parker, C.; Pearse, M.; Richaud, P.; Sargos, P.; Sydes, M.R.; Brawley, C.; Brihoum, M.; et al. Adjuvant or early salvage radiotherapy for the treatment of localised and locally advanced prostate cancer: A prospectively planned systematic review and meta-analysis of aggregate data. Lancet 2020, 396, 1422-1431. [CrossRef]

2. Parker, C.C.; Clarke, N.W.; Cook, A.D.; Kynaston, H.G.; Petersen, P.M.; Catton, C.; Cross, W.; Logue, J.; Parulekar, W.; Payne, H.; et al. Timing of radiotherapy after radical prostatectomy (RADICALS-RT): A randomized controlled phase 3 trial. Lancet 2020, 396, 1413-1421. [CrossRef]

3. Sargos, P.; Chabaud, S.; Latorzeff, I.; Magné, N.; Benyoucef, A.; Supiot, S.; Pasquier, D.; Abdiche, M.S.; Gilliot, O.; Graff-Cailleaud, P.; et al. Adjuvant radiotherapy versus early salvage radiotherapy plus short-term androgen deprivation therapy in men with localised prostate cancer after radical prostatectomy (GETUG-AFU 17): A randomised, phase 3 trial. Lancet Oncol. 2020, 21, 1341-1352. [CrossRef]

4. Kneebone, A.; Fraser-Browne, C.; Duchesne, G.M.; Fisher, R.; Frydenberg, M.; Herschtal, A.; Williams, S.G.; Brown, C.; Delprado, W.; Haworth, A.; et al. Adjuvant radiotherapy versus early salvage radiotherapy following radical prostatectomy (TROG 08.03/ANZUP RAVES): A randomised, controlled, phase 3, non-inferiority trial. Lancet Oncol. 2020, 21, 1331-1340. [CrossRef]

5. Nyarangi-Dix, J.N.; Steimer, J.; Bruckner, T.; Jakobi, H.; Koerber, S.A.; Hadaschik, B.; Debus, J.; Hohenfellner, M. Postprostatectomy radiotherapy adversely affects urinary continence irrespective of radiotherapy regime. World J. Urol. 2017, 35, 1841-1847. [CrossRef]

6. Ávila, M.; Becerra, V.; Guedea, F.; Suárez, J.F.; Fernandez, P.; Macías, V.; Mariño, A.; Hervás, A.; Herruzo, I.; Ortiz, M.J.; et al. Estimating preferences for treatments in patients with localized prostate cancer. Int. J. Radiat. Oncol. 2015, 91, 277-287. [CrossRef]

7. Hoffman, R.M.; Lo, M.; Clark, J.A.; Albertsen, P.C.; Barry, M.J.; Goodman, M.; Penson, D.F.; Stanford, J.L.; Stroup, A.M.; Hamilton, A.S. Treatment decision regret among long-term survivors of localized prostate cancer: Results from the prostate cancer outcomes study. J. Clin. Oncol. 2017, 35, 2306-2314. [CrossRef] [PubMed]

8. Tendulkar, R.D.; Agrawal, S.; Gao, T.; Efstathiou, J.A.; Pisansky, T.M.; Michalski, J.M.; Koontz, B.F.; Hamstra, D.A.; Feng, F.Y.; Liauw, S.L.; et al. Contemporary update of a multi-institutional predictive nomogram for salvage radiotherapy after radical prostatectomy. J. Clin. Oncol. 2016, 34, 3648-3654. [CrossRef] [PubMed]

9. Fiorino, C.; Broggi, S.; Fossati, N.; Cozzarini, C.; Goldner, G.; Wiegel, T.; Hinkelbein, W.; Karnes, R.J.; Boorjian, S.A.; Haustermans, K.; et al. Predicting the 5-year risk of biochemical relapse after postprostatectomy radiation therapy in $\geq$ PT2, pN0 patients with a comprehensive tumor control probability model. Int. J. Radiat. Oncol. 2016, 96, 333-340. [CrossRef] 
10. Thompson, I.M.; Valicenti, R.K.; Albertsen, P.C.; Davis, B.; Goldenberg, S.L.; Hahn, C.; Klein, E.A.; Michalski, J.M.; Roach, M.; Sartor, O.; et al. Adjuvant and salvage radiotherapy after prostatectomy: AUA/ASTRO guideline. J. Urol. 2013, 190, 441-449. [CrossRef]

11. Suardi, N.; Gallina, A.; Lista, G.; Gandaglia, G.; Abdollah, F.; Capitanio, U.; Dell'Oglio, P.; Nini, A.; Salonia, A.; Montorsi, F.; et al. Impact of adjuvant radiation therapy on urinary continence recovery after radical prostatectomy. Eur. Urol. 2014, 65, 546-551. [CrossRef]

12. Zaffuto, E.; Gandaglia, G.; Fossati, N.; Dell'Oglio, P.; Moschini, M.; Cucchiara, V.; Suardi, N.; Mirone, V.; Bandini, M.; Shariat, S.F.; et al. Early postoperative radiotherapy is associated with worse functional outcomes in patients with prostate cancer. J. Urol. 2017, 197, 669-675. [CrossRef] [PubMed]

13. van Stam, M.A.; Aaronson, N.K.; Pos, F.J.; Bosch, J.R.; Kieffer, J.M.; Tillier, C.N.; van der Poel, H.G. The effect of salvage radiotherapy and its timing on the health-related quality of life of prostate cancer patients. Eur. Urol. 2016, 70, 751-757. [CrossRef] [PubMed]

14. Adam, M.; Tennstedt, P.; Lanwehr, D.; Tilki, D.; Steuber, T.; Beyer, B.; Thederan, I.; Heinzer, H.; Haese, A.; Salomon, G.; et al. Functional outcomes and quality of life after radical prostatectomy only versus a combination of prostatectomy with radiation and hormonal therapy. Eur. Urol. 2017, 71, 330-336. [CrossRef] [PubMed]

15. Pinkawa, M.; Fischedick, K.; Asadpour, B.; Gagel, B.D.; Piroth, M.D.; Holy, R.; Krenkel, B.; Eble, M.J. Health-related quality of life after adjuvant and salvage postoperative radiotherapy for prostate cancer-A prospective analysis. Radiother. Oncol. 2008, 88, 135-139. [CrossRef] [PubMed]

16. Sowerby, R.J.; Gani, Y.; Yim, H.; Radomsky, S.B.; Catton, C. Long-term complications in men who have early or late radiotherapy after radical prostatectomy. Can. Urol. Assoc. J. 2014, 8, 253-258. [CrossRef] [PubMed]

17. Vogel, M.M.E.; Kessel, K.A.; Gschwend, J.E.; Weichert, W.; Wilkens, J.J.; Combs, S.E. Early and late toxicity profiles of patients receiving immediate postoperative radiotherapy versus salvage radiotherapy for prostate cancer after prostatectomy. Strahlenther. Onkol. 2018, 195, 131-144. [CrossRef]

18. Donovan, J.L.; Hamdy, F.C.; Lane, J.A.; Mason, M.; Metcalfe, C.; Walsh, E.; Blazeby, J.M.; Peters, T.J.; Holding, P.; Bonnington, S.; et al. Patient-reported outcomes after monitoring, surgery, or radiotherapy for prostate cancer. N. Engl. J. Med. 2016, 375, 1425-1437. [CrossRef] [PubMed]

19. Budäus, L.; Bolla, M.; Bossi, A.; Cozzarini, C.; Crook, J.; Widmark, A.; Wiegel, T. Functional outcomes and complications following radiation therapy for prostate cancer: A critical analysis of the literature. Eur. Urol. 2012, 61, 112-127. [CrossRef]

20. Sini, C.; Chiorda, B.N.; Gabriele, P.; Sanguineti, G.; Morlino, S.; Badenchini, F.; Cante, D.; Carillo, V.; Gaetano, M.; Giandini, T.; et al. Patient-reported intestinal toxicity from whole pelvis intensity-modulated radiotherapy: First quantification of bowel dose-volume effects. Radiother. Oncol. 2017, 124, 296-301. [CrossRef]

21. Sini, C.; Fiorino, C.; Perna, L.; Chiorda, B.N.; Deantoni, C.L.; Bianchi, M.; Sacco, V.; Briganti, A.; Montorsi, F.; Calandrino, R.; et al. Dose-volume effects for pelvic bone marrow in predicting hematological toxicity in prostate cancer radiotherapy with pelvic node irradiation. Radiother. Oncol. 2016, 118, 79-84. [CrossRef]

22. Cozzarini, C.; Chiorda, B.N.; Sini, C.; Fiorino, C.; Briganti, A.; Montorsi, F.; Di Muzio, N. Hematologic toxicity in patients treated with postprostatectomy whole-pelvis irradiation with different intensity modulated radiation therapy techniques is not negligible and is prolonged: Preliminary results of a longitudinal, observational study. Int. J. Radiat. Oncol. 2016, 95, 690-695. [CrossRef]

23. Avery, K.; Donovan, J.; Peters, T.J.; Shaw, C.; Gotoh, M.; Abrams, P. ICIQ: A brief and robust measure for evaluating the symptoms and impact of urinary incontinence. Neurourol. Urodyn. 2004, 23, 322-330. [CrossRef] [PubMed]

24. Francis, L.J.; Brown, L.B.; Philipchalk, R. The development of an abbreviated form of the revised Eysenck personality questionnaire (EPQR-A): Its use among students in England, Canada, the U.S.A. and Australia. Pers. Individ. Differ. 1992, 13, 443-449. [CrossRef]

25. Landoni, V.; Fiorino, C.; Cozzarini, C.; Sanguineti, G.; Valdagni, R.; Rancati, T. Predicting toxicity in radiotherapy for prostate cancer. Phys. Med. 2016, 32, 521-532. [CrossRef] [PubMed]

26. Cozzarini, C.; Rancati, T.; Palorini, F.; Avuzzi, B.; Garibaldi, E.; Balestrini, D.; Cante, D.; Munoz, F.; Franco, P.; Girelli, G.; et al. Patient-reported urinary incontinence after radiotherapy for prostate cancer: Quantifying the dose-effect. Radiother. Oncol. 2017, 125, 101-106. [CrossRef]

27. Barry, M.J.; Gallagher, P.M.; Skinner, J.S.; Fowler, F.J. Adverse effects of robotic-assisted laparoscopic versus open retropubic radical prostatectomy among a nationwide random sample of medicare-age men. J. Clin. Oncol. 2012, 30, 513-518. [CrossRef] [PubMed]

28. Nyberga, M.; Hugossonc, J.; Wiklund, P.; Sjoberg, D.; Wilderäng, U.; Carlsson, S.V.; Carlsson, S.; Stranne, J.; Steineck, G.; Haglind, E.; et al. Functional and oncologic outcomes between open and robotic radical prostatectomy at 24-month follow-up in the Swedish LAPPRO trial. Eur. Urol. Oncol. 2018, 1, 353-360. [CrossRef] [PubMed]

29. Ávila, M.; Patel, L.; López, S.; Cortes-Sanabría, L.; Garín, O.; Pont, À.; Ferrer, F.; Boladeras, A.; Zamora, V.; Fosså, S.; et al. Patient-reported outcomes after treatment for clinically localized prostate cancer: A systematic review and meta-analysis. Cancer Treat. Rev. 2018, 66, 23-44. [CrossRef]

30. Leufgens, F.; Berneking, V.; Vögeli, T.-A.; Kirschner-Hermanns, R.; Eble, M.J.; Pinkawa, M. Quality of life changes $>10$ years after postoperative radiation therapy after radical prostatectomy for prostate cancer. Int. J. Radiat. Oncol. 2019, 105, 382-388. [CrossRef] 